\title{
Does the 5-2-1 criteria identify patients with advanced Parkinson's disease? Real- world screening accuracy and burden of 5-2-1-positive patients in 7 countries
}

Irene A. Malaty ${ }^{1 *}$, Pablo Martinez-Martin², K. Ray Chaudhuri ${ }^{3}$, Per Odin ${ }^{4}$, Matej Skorvanek ${ }^{5,6}$, Joohi Jimenez-Shahed ${ }^{7}$, Michael J. Soileau ${ }^{8}$, Susanna Lindvall ${ }^{9}$, Josefa Domingos ${ }^{9,10}$, Sarah Jones ${ }^{11}$, Ali Alobaidi ${ }^{12,13}$, Yash J. Jalundhwala ${ }^{12}$, Prasanna L. Kandukuri ${ }^{12}$, Koray Onuk ${ }^{12}$, Lars Bergmann ${ }^{12}$, Samira Femia ${ }^{12}$, Michelle Y. Lee ${ }^{12}$, Jack Wright ${ }^{14}$ and Angelo Antonini ${ }^{15}$

\begin{abstract}
Background: The burden of Parkinson's disease (PD) worsens with disease progression. However, the lack of objective and uniform disease classification challenges our understanding of the incremental burden in patients with advanced Parkinson's disease (APD) and suboptimal medication control. The 5-2-1 criteria was proposed by clinical consensus to identify patients with advancing PD. Our objective was to evaluate the screening accuracy and incremental clinical burden, healthcare resource utilization (HCRU), and humanistic burden in PD patients meeting the 5-2-1 screening criteria.

Methods: Data were drawn from the Adelphi Parkinson's Disease Specific Program (DSP ${ }^{\mathrm{TM}}$ ), a multi-country pointin-time survey (2017-2020). People with PD who were naive to device-aided therapy and on oral PD therapy were included. Patients meeting the 5-2-1 screening criteria had one or more of the three clinical indicators of APD: (i) $\geq 5$ doses of oral levodopa/day, OR (ii) "off" symptoms for $\geq 2 \mathrm{~h}$ of waking day, OR (iii) $\geq 1 \mathrm{~h}$ of troublesome dyskinesia. Clinician assessment of PD stage was used as the reference in this study. Clinical screening accuracy of the 5-2-1 criteria was assessed using area under the curve and multivariable logistic regression models. Incremental clinical, HCRU, and humanistic burden were assessed by known-group comparisons between 5 and 2-1-positive and negative patients.

Results: From the analytic sample $(n=4714), 33 \%$ of patients met the 5-2-1 screening criteria. Among physicianclassified APD patients, 78.6\% were 5-2-1 positive. Concordance between clinician judgment and 5-2-1 screening criteria was > 75\%. 5-2-1-positive patients were nearly 7-times more likely to be classified as APD by physician judgment. Compared with the 5-2-1-negative group, 5-2-1-positive patients had significantly higher clinical, HCRU, and humanistic burden across all measures. In particular, 5-2-1-positive patients had 3.8-times more falls, 3.6-times higher annual hospitalization rate, and 3.4-times greater dissatisfaction with PD treatment. 5-2-1-positive patients also had significantly lower quality of life and worse caregiver burden.
\end{abstract}

\footnotetext{
*Correspondence: Irene.malaty@neurology.ufl.edu

${ }^{1}$ University of Florida, Fixel Institute for Neurological Diseases, Gainesville,

FL, USA

Full list of author information is available at the end of the article
}

(c) The Author(s) 2022. Open Access This article is licensed under a Creative Commons Attribution 4.0 International License, which permits use, sharing, adaptation, distribution and reproduction in any medium or format, as long as you give appropriate credit to the original author(s) and the source, provide a link to the Creative Commons licence, and indicate if changes were made. The images or other third party material in this article are included in the article's Creative Commons licence, unless indicated otherwise in a credit line to the material. If material is not included in the article's Creative Commons licence and your intended use is not permitted by statutory regulation or exceeds the permitted use, you will need to obtain permission directly from the copyright holder. To view a copy of this licence, visit http://creativecommons.org/licenses/by/4.0/. The Creative Commons Public Domain Dedication waiver (http://creativeco mmons.org/publicdomain/zero/1.0/) applies to the data made available in this article, unless otherwise stated in a credit line to the data. 
Conclusions: 5-2-1 criteria demonstrated potential as a screening tool for identifying people with APD with considerable clinical, humanistic, and HCRU burden. The 5-2-1 screening criteria is an objective and reliable tool that may aid the timely identification and treatment optimization of patients inadequately controlled on oral PD medications.

Keywords: Advanced Parkinson's disease, 5-2-1 criteria, Screening performance, clinical burden

\section{Background}

Parkinson's disease (PD) is one of the fastest growing neurological disorders worldwide, with a reported $118 \%$ increase in prevalence between 1990 and 2015 [1, 2]. In 2015, approximately 6.2 million individuals globally were estimated to have PD $[1,2]$.

As PD progresses, patients tend to experience greater disability and an impaired ability to perform activities of daily living (ADLs) [3, 4], as well as fluctuating response to standard oral PD therapies [4] and delayed gastric emptying [5] leading to poorer symptom control. In order to achieve optimal PD symptom control, patients may require advanced treatment options such as deep brain stimulation, subcutaneous apomorphine infusion, and levodopa-carbidopa intestinal gel (LCIG) [6].

Unfortunately, progress in APD management has been impeded by a lack of objective and standardized diagnostic criteria to identify patients with advanced disease in clinical practice. This is further compounded by the complexity and heterogeneity of PD symptoms, making timely and accurate patient identification even more challenging [4]. A uniform set of criteria for rapidly identifying APD is therefore urgently required to screen for patients with suboptimal symptom control [4, 6-8].

A number of different screening and management approaches have previously been used in an attempt to identify APD, including the use of biomarkers $[9,10]$ and uni- and multi-dimensional scales such as the Hoehn and Yahr (H\&Y) scale [11], the Parkinson Disease Composite Scale (PDCS) [12], and the Cuestionario De Enfermedad de Parkinson Avanzada (Questionnaire for Advanced Parkinson's Disease; CDEPA) [13]. In addition, the Unified Parkinson's Disease Rating Scale (UPDRS) is widely used to follow the course of PD [14], but cannot be used to truly diagnose APD as outcomes may vary according to the time of the clinical evaluation (i.e., whether the patient was in the ON or OFF state).

An alternative approach to screening for APD has recently emerged from a consensus group tasked with assessing clinical indicators of APD $[4,15]$ in a modified Delphi study. The consensus group agreed on 15 clinically relevant indicators (six motor symptoms [MS], five non-motor symptoms [NMS], and four functional impairments) for suspected APD [4]. Of the 15 identifiers described, a combination of three key criteria was later proposed as a simple-to-use screening tool to identify patients with suspected APD [4, 16, 17], namely: i) taking $\geq 5$ doses of oral levodopa per day, or ii) having 'off' symptoms for $\geq 2 \mathrm{~h}$ of waking day, or iii) having $\geq 1 \mathrm{~h}$ of troublesome dyskinesia per waking day. Under this proposal, patients meeting at least one of these three screening criteria ('5-2-1 positive' patients) would be considered as potentially having advanced disease.

Previous studies have suggested the potential utility of the 5-2-1 criteria as a screening tool for APD [17, 18]. In one study, individuals meeting that 5-2-1 criteria were found to have significantly poorer health outcomes, including worse quality of life (QoL), greater NMS burden, and greater caregiver burden [16]. In a retrospective analysis of the DUOGLOBE study, $98 \%$ of patients who had been selected for LCIG by investigators were found to fulfill at least one of the 5-2-1 criteria, suggesting that the 5-2-1 criteria apply to a population of patients identified by clinicians as having APD [19].

However, the few studies evaluating the 5-2-1 screening criteria have tended to be small, single-centered investigations offering limited evidence on real-world screening accuracy of the 5-2-1 criteria and little information on the incremental burden experienced by 5-2-1-positive patients [18]. To improve our understanding of the utility of the 5-2-1 screening criteria in every day clinical practice, the current real-world analysis was undertaken. The objectives of this study were two-fold: to evaluate the screening accuracy of the 5-2-1 criteria in identifying people with APD, and to quantify the incremental clinical and humanistic burden as well as the healthcare resource utilization (HCRU) of 5-2-1-positive patients.

\section{Methods}

Data for this retrospective study were drawn from the Adelphi Parkinson's Disease Specific Program $\left(\mathrm{DSP}^{\mathrm{TM}}\right)$, a point-in-time survey of physicians and their consulting PD patients presenting in a real-world clinical setting. The DSP was conducted in G7 countries (France, Germany, Italy, Japan, Spain, the UK, and the USA) between 2017 and 2020. The DSP methodology has been previously published and validated [20]. Participating physicians completed a record form for the next 12 consecutively consulting patients meeting the eligibility criteria; the physician-reported questionnaire form contained questions on patients' demographics, clinical 
assessments, clinical outcomes, medication use and history, healthcare resource utilization, and concomitant conditions. Each patient for whom the physician completed a form was then invited to complete a patientreported questionnaire, which recorded demographics, current condition, level of satisfaction with their treatment, and QoL. If the patient had a caregiver, they were also invited to complete a form, reporting on demographics, and burden of care.

Respondents provided informed consent for use of their data; all data were aggregated and de-identified before receipt.

\section{Participants}

Physicians were eligible to participate in the survey if they were a neurologist, and they were personally responsible for treatment decisions and management of patients with PD. Patients were eligible for inclusion in the survey if they were diagnosed with PD on or before the date of their last consultation (i.e., at the time of data collection), and aged $\geq 18$ years. Patients were eligible for inclusion in this analysis if they were taking oral therapy for PD, and naive to treatment with device-aided therapy (DAT).

\section{Measures}

Patients meeting the 5-2-1 screening criteria were identified as those experiencing one or more of the following: (i) taking $\geq 5$ doses of oral levodopa per day, OR (ii) having 'off' symptoms for $\geq 2 \mathrm{~h}$ of waking day, OR (iii) having $\geq 1 \mathrm{~h}$ of troublesome dyskinesia per waking day. The physician's opinion of disease severity (defined as APD vs non-advanced PD), based on overall patient history including demographics, clinical characteristic, concomitant medications, and other patient-reported outcomes, was used as the reference for this study. As all participating physicians had to see a minimum of three PD patients per week, all were considered highly experienced and can reasonably be expected to reliably distinguish degree of PD severity.

We also captured clinical, HCRU, and humanistic PD burden to quantify the burden of patients meeting the 5-2-1 screening criteria. Clinical burden was evaluated based on the following measures: UPDRS (clinical rating scale for PD encompassing behavior and mood, ADLs, MS, and complications; higher score indicates greater burden) [21], Mini-Mental State Examination (MMSE; 11-question instrument testing five areas of cognitive function; lower score indicates worse cognitive function) [22], Charlson comorbidity index (based on a number of conditions that are each assigned an integer weight from one to six; higher score indicates greater comorbidity burden) [23], ADL (assessed as percentage of patients able to carry out specific ADLs independently), and falls (mean number of PD-related falls in the last 12 months, based on patient report). HCRU was assessed by considering the following measures: hospitalization rate (number and percentage of patients hospitalized in the last 12 months for $\mathrm{PD}$, including surgical procedures), physician consultations (number of consultations with a physician in the last 12 months, as captured on patient records), and hours of caregiver help (total number of hours of caregiver help required per week, including professional and non-professional support). Humanistic burden was based on following measures: Parkinson's Disease Questionnaire (PDQ-39; questionnaire assessing PD-related difficulties in daily living across eight dimensions; higher score indicates greater burden) [24], EuroQol 5 Dimension (EQ-5D; a generic instrument to assess health-related QoL; scores range from 0 to 1 , with a lower score indicating poorer QoL) [25], PD treatment satisfaction (number and percentage of patients reporting satisfaction with their drug treatment [measured on a patient-rated 7-point scale] with their drug treatment), and Zarit Burden Interview (ZBI; caregiver self-report 29-item questionnaire; higher score indicates greater burden) [26].

\section{Statistical analysis}

Multivariable logistic regression models were computed to evaluate the screening performance of the 5-2-1 criteria as a predictor and clinician assessment of APD as an outcome. The screening performance of the 5-2-1 criteria was evaluated based on the following: odds ratio (OR) and 95\% confidence interval (CI), correct classification percentage, and area under the curve (AUC). Correct classification is the percentage of patients correctly classified per 5-2-1 criteria (sum of true positive and true negatives divided by total number of patients), and is described using the term 'concordance'. AUC is a screening accuracy measure that balances sensitivity (i.e., presence of the 5-2-1 screening criteria in patients with APD according to physician's judgment) and specificity (i.e., absence of the 5-2-1 screening criteria in patients with non-advanced PD). AUC is interpreted as follows: $\mathrm{AUC}=0.5$ suggests a non-informative result (no better than chance); $\mathrm{AUC}=0.5$ to $\leq 0.7$ is considered less accurate; $\mathrm{AUC}=0.7$ to $\leq 0.9$ is considered moderately accurate, AUC 0.9 to $<1$ is considered highly accurate, and $A U C=1$ is considered the perfect test [27-29]. All logistic regression models were adjusted for potential confounders including country, patient age $(<65$ years or $\geq 65$ years), gender, time since PD diagnosis, and Charlson comorbidity index.

Incremental clinical burden, healthcare resource utilization, and humanistic burden was evaluated based on known-group comparisons between 5 and 2-1 positive 
and 5-2-1-negative groups using Wilcoxon-MannWhitney, t-test, chi-square, and Fisher's exact tests, as appropriate.

\section{Results}

\section{Sample characteristics}

In total, 4714 patients were included from all G7 countries (France: $10.5 \%$, Germany: $12.6 \%$, Italy: 11.3\%, Japan: $11.9 \%$, Spain: $11.6 \%$, UK: $12.4 \%$, and USA: $29.6 \%)$. The mean (standard deviation, SD) time since diagnosis was 4.3 (4.4) years, and $33.5 \%$ of patients were H\&Y stage $3-5$ in the "on" state. Of the 4714 patients included, $14.9 \%$ ( $n=702$ ) were classified by the physician as having APD.

According to the criteria described above, $32.8 \%$ of patients $(n=1546)$ were $5-2-1$ positive, including 552 patients who had been classified by the physician as having APD (78.6\% of all APD patients). Compared with 5-2-1-negative patients, 5-2-1-positive patients were older, had a longer time since diagnosis, had a higher H\&Y stage, and were more often classified as having APD by their physician (Table 1 ). 5-2-1 positive patients had a higher Charlson Comorbidity Index, the observed difference being informed by a greater prevalence of cardiovascular conditions, diabetes, and dementia (Supplementary Table 1).

\section{Accuracy}

An evaluation of the screening accuracy of the 5-2-1 criteria and clinical indicators of APD with respect to the physician diagnosis is summarized in Table 2. The 5-2-1 screening criteria demonstrated moderate screening accuracy for APD, with AUC values $\geq 0.80$. A comparable analysis carried out using $H \& Y$ stages (H\&Y stage $1-2$ vs H\&Y stage 3-5) in place of physician judgement, and the outcome was similar (moderate screening accuracy for APD) (Supplementary Table 2).

From the multivariable logistic regression, 5-2-1-positive patients were nearly 7 times more likely to be classified as having APD than 5-2-1-negative patients (adjusted OR 6.84; 95\% CI, 5.50-8.51). Patients taking $\geq 5$ oral levodopa doses/day were over 3 times more likely to be classified as having APD, patients having $\geq 2 \mathrm{~h}$ of 'off'time/waking day were over 7 times as likely, and patients having $\geq 1 \mathrm{~h}$ of troublesome dyskinesia/waking day were 5 times more likely to be classified as having APD.

There was a minority of patients for whom clinician judgment and 5-2-1 screening criteria did not align, with 150 (3.2\%) 5-2-1-negative patients classified as advanced PD and 994 (21.1\%) 5-2-1-positive patients classified as non-advanced PD by the clinician. However, overall concordance was greater than $75 \%$. Further

Table 1 Clinical characteristics of 5-2-1-positive and 5-2-1-negative patients

\begin{tabular}{|c|c|c|c|c|}
\hline & All patients $(n=4714)^{a}$ & 5-2-1 positive $(n=1546)^{a}$ & $5-2-1$ negative $(n=3168)^{a}$ & $P$-value \\
\hline \multicolumn{5}{|l|}{ Patient age } \\
\hline Mean (SD) & $69.3(10.6)$ & 71.7 (10.4) & $68.2(10.5)$ & $<0.0001$ \\
\hline$\geq 65$ years, $n(\%)$ & $3309(70.2)$ & $1231(79.6)$ & $2078(65.6)$ & $<0.0001$ \\
\hline \multicolumn{5}{|l|}{ Gender, n (\%) } \\
\hline Male & $2866(60.8)$ & $964(62.4)$ & $1902(60.0)$ & 0.1274 \\
\hline Charlson Comorbidity Index, mean (SD) & $0.4(1.0)$ & $0.6(1.2)$ & $0.3(0.9)$ & $<0.0001$ \\
\hline \multicolumn{5}{|l|}{ Charlson Comorbidity Index, n (\%) } \\
\hline None (score 0) & $3765(79.9)$ & $1108(71.7)$ & $2657(83.9)$ & $<0.0001$ \\
\hline Mild (score 1-2) & $787(16.7)$ & $357(23.1)$ & $430(13.6)$ & \\
\hline Moderate (score 3-4) & $120(2.5)$ & $62(4.0)$ & $58(1.8)$ & \\
\hline Severe (score $5+$ ) & $42(0.9)$ & $19(1.2)$ & $23(0.7)$ & \\
\hline Time since PD diagnosis (years), mean (SD) & $4.3(4.4)(n=3712)$ & $7.2(5.1)(n=1162)$ & $3.0(3.2)(n=2550)$ & $<0.0001$ \\
\hline \multicolumn{5}{|l|}{ Physician judgment of PD stage, n (\%) } \\
\hline Non-advanced & $4012(85.1)$ & $994(64.3)$ & $3018(95.3)$ & $<0.0001$ \\
\hline Advanced & $702(14.9)$ & $552(35.7)$ & $150(4.7)$ & \\
\hline \multicolumn{5}{|l|}{ Hoehn and Yahr stage, n (\%) } \\
\hline 1 & $1593(33.8)$ & $1579(39.4)$ & $14(2.0)$ & $<0.0001$ \\
\hline 2 & $1545(32.8)$ & $1492(37.2)$ & $53(7.5)$ & \\
\hline 3 & $932(19.8)$ & $778(19.4)$ & $154(21.9)$ & \\
\hline 4 & $457(9.7)$ & $147(3.7)$ & $310(44.2)$ & \\
\hline 5 & $187(4.0)$ & $16(0.4)$ & $171(24.4)$ & \\
\hline
\end{tabular}

PD Parkinson's disease, SD Standard deviation

a Patient number unless otherwise stated 
Table 2 Screening accuracy of the 5-2-1 screening criteria in identifying patients with advanced Parkinson's disease

\begin{tabular}{|c|c|c|c|c|c|}
\hline \multirow[b]{2}{*}{ Indicator } & \multicolumn{2}{|c|}{$\begin{array}{l}\text { Advanced } \\
\text { PD }\end{array}$} & \multicolumn{3}{|c|}{ Adjusted Model $^{\mathrm{a}}$} \\
\hline & No & Yes & OR $(95 \% \mathrm{Cl})$ & $\begin{array}{l}\text { Correct } \\
\text { Classification } \\
(\%)^{b}\end{array}$ & AUC $^{c}$ \\
\hline
\end{tabular}

\begin{tabular}{|c|c|c|c|c|c|}
\hline \multicolumn{6}{|c|}{ 5-2-1 screening criteria } \\
\hline Negative & 3018 & 150 & 1 & - & - \\
\hline Positive & 994 & 552 & $6.84(5.50,8.51)$ & 88.1 & 0.89 \\
\hline \multicolumn{6}{|c|}{ Individual clinical indicators } \\
\hline \multicolumn{6}{|c|}{$\geq 2 \mathrm{~h}$ off-time/day } \\
\hline Negative & 3299 & 208 & 1 & - & - \\
\hline Positive & 713 & 494 & $7.07(5.76,8.68)$ & 88.4 & 0.89 \\
\hline \multicolumn{6}{|c|}{$\geq 1 \mathrm{~h}$ troublesome dyskinesia/day } \\
\hline Negative & 3944 & 591 & 1 & - & - \\
\hline Positive & 68 & 111 & $5.56(3.85,8.02)$ & 87.1 & 0.85 \\
\hline \multicolumn{6}{|c|}{$\geq 5$ doses of oral levodopa/day } \\
\hline Negative & 3501 & 413 & 1 & - & - \\
\hline Positive & 511 & 289 & $3.03(2.45,3.73)$ & 87.1 & 0.86 \\
\hline
\end{tabular}

${ }^{a}$ Regressions adjusted for age, sex, time since diagnosis, Charlson Comorbidity Index, and country. ${ }^{b}$ Correct classification is the percentage of patients correctly classified per 5-2-1 criteria (sum of true positive and true negatives divided by total number of patients). ${ }^{C}$ AUC is a screening accuracy measure that balances sensitivity and specificity. AUC is interpreted as follows: $A U C=0.5$, non-informative; $A U C=0.5$ to $\leq 0.7$, less accurate; $A U C=0.7$ to $\leq 0.9$, moderately accurate, $A \cup C 0.9$ to $<1$, highly accurate; $A U C=1$, perfect test [27-29]

AUC Area under the curve, $\mathrm{CI}$ Confidence interval, OR Odds ratio

analysis revealed that the small group of false negatives (clinician-defined APD patients who were 5-2-1 negative) tended to be older, diagnosed at a later age, and had higher trend for NMS compared with 5-2-1-positive patients classified as having non-advanced disease (Supplementary Table 3).

\section{Clinical burden}

Clinical burden across all measures assessed was significantly greater in the 5-2-1-postive group compared with 5-2-1-negative patients (Fig. 1). In particular, 5-2-1-positive patients had higher UPDRS total score $(\Delta+15.7$ [41.2 vs $25.5 ; p<0.0001])$ and lower MMSE score $(\Delta-1.8$ [23.4 vs $25.2 ; p<0.0001])$, indicating a greater clinical burden compared with 5-2-1-negative patients. Patients in the 5-2-1-positive group had experienced more PD-related falls in the last 12 months $(\Delta$ +1.7 falls [2.3 vs 0.6 falls; $p<0.0001]$ ) compared with 5-2-1-negative patients. Significantly fewer 5-2-1-positive patients showed independence in carrying out ADLs $(24.3 \%$ vs. $56.2 \% ; p<0.0001)$. Similar findings were seen when data were analyzed according to physician judgement (Fig. 2) or H\&Y stage (Supplementary Fig. 1).

\section{Healthcare resource utilization burden}

Healthcare resource utilization was significantly greater among 5-2-1-positive patients than 5-2-1-negative patients across all measures assessed (Fig. 3). In particular, considerably more patients in the 5-2-1-positive group $(19.3 \%$ vs $5.4 \% ; p<0.0001)$ were hospitalized in the last 12 months. 5-2-1-positive patients had a significantly higher number of annual physician consultations $(\Delta+3.3$ consults/year [8.3 vs 5.0 consults/year; $p<0.0001]$ ) and required a significantly greater amount of professional and non-professional caregiver support $(\Delta+19.7 \mathrm{~h} /$ week overall [30.8 vs $11.1 \mathrm{~h} /$ week; $p<0.0001]$ ). Similar findings were seen when data were analyzed according to physician judgement (Fig. 4) or H\&Y stage (Supplementary Fig. 2).

\section{Humanistic burden}

Humanistic burden across all measures assessed was significantly worse among 5-2-1-positive patients (Fig. 5). Compared with the 5-2-1-negative group, 5-2-1-positive patients had a significantly higher PDQ-39 index score $(\Delta+10.5$ [33.6 vs $23.1 ; p<0001])$ and lower EQ-5D utility score $(\Delta-0.16[0.53$ vs $0.69 ; p<0.0001])$, indicating a greater burden on PD and health-related QoL among 5-2-1-positive patients. A significantly greater proportion of patients in the 5-2-1-positive group were reported to be unsatisfied with their drug treatment compared with $5-2-1$-negative patients $(14.9 \%$ vs $4.4 \%$; $p<0.0001)$. Caregivers of 5-2-1-positive patients also demonstrated worse caregiver burden based on ZBI total score $(\Delta+8.8$ [32.0 vs $23.3 ; p<0.0001])$. Similar findings were seen when data were analyzed according to physician judgement (Fig. 6) or H\&Y stage (Supplementary Fig. 3).

\section{Discussion}

To date, this is the largest study to have evaluated the performance of the 5-2-1 criteria in screening and evaluating patients with APD. The 5-2-1 screening criteria demonstrated potential as a screening tool for identifying people with APD. Patients classified as 5-2-1-positive while receiving oral PD treatment had incrementally higher clinical, humanistic, and HCRU burden compared with the 5-2-1-negative group. Our data may suggest the potential of the 5-2-1 criteria as a screening tool to help recognize people with APD who are sub-optimally controlled on oral PD treatment in the real-world $[16,19]$. Our results also reinforce the need to identify current unmet needs among these patients due to their increased disease burden.

Although effective disease management is key at all stages of PD, the need for greater individual 
UPDRS score

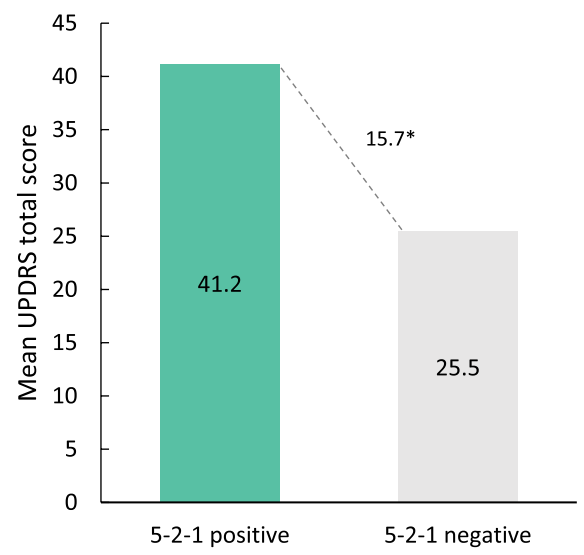

Falls

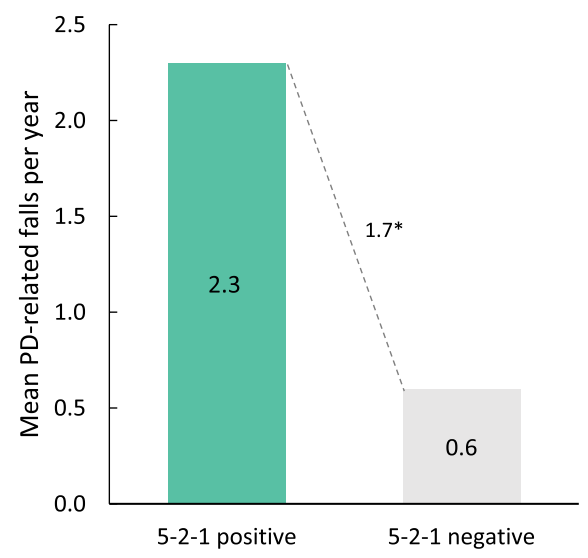

MMSE score

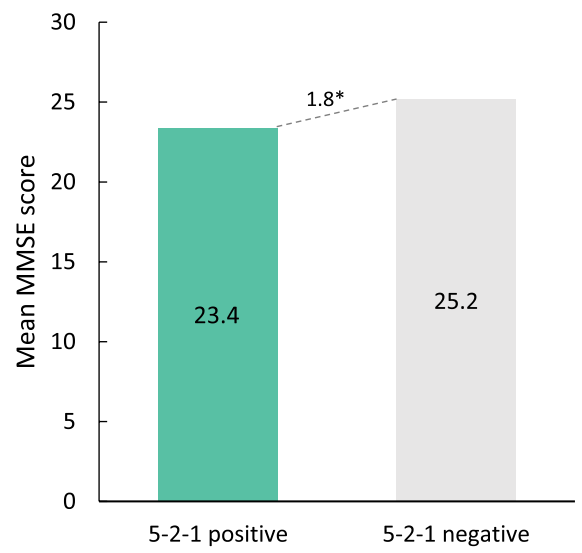

ADL independence

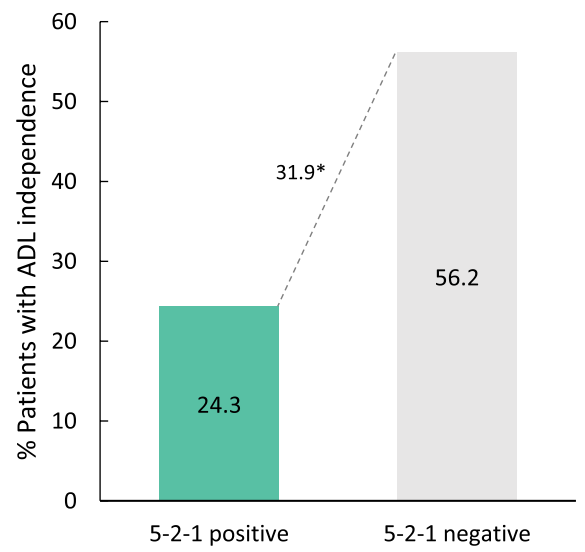

Fig. 1 Measures of clinical burden in 5-2-1-positive patients compared with 5-2-1-negative patients. ${ }^{*} p<0.0001 .5-2-1$, taking $\geq 5$ doses of oral levodopa/day, OR having $\geq 2$ h 'off'-time/waking day, OR having $\geq 1 \mathrm{~h}$ troublesome dyskinesia/waking day. ADL, activities of daily living; MMSE, Mini-Mental State Examination; PD, Parkinson's disease; UPDRS, Unified Parkinson's Disease Rating Scale

customization becomes increasingly important as the disease advances [30]. A number of previous attempts have been made to devise simple screening tools that can rapidly identify people with APD who would benefit from further monitoring and optimization of their care plan. In addition, management tools have been developed to evaluate PD symptoms, guide treatment protocols, and ensure that patients with the greatest burden receive optimal treatment. In terms of rapid screening for APD, the duration of disease has traditionally been the first characteristic to be considered. However, definitions of 'long' disease duration vary among studies [31-34]. Symptoms do not progress linearly or uniformly over time, and deteriorate at a different pace in different patients. For these reasons, a long duration of disease does not necessarily correspond to more advanced disease [35]. Tools designed to guide the management of PD have tended to include instruments based on specific clinical criteria $[10,13,15,16]$, the use of ratings scales such as the H\&Y or UPDRS [14, 36], and the use of biomarkers [37]. However, the universal application of these tools is limited by the substantial time and resources required to apply them.

New approaches have been developed over recent years as we recognized the clear need for improved screening and management tools. One example is an algorithm that was devised to screen for APD based on any 30-day average levodopa equivalent dose (LED) $>1000 \mathrm{mg} /$ day [3]. When this algorithm was applied, approximately $20 \%$ of the sample was classified as having advanced disease. However, this algorithm is specific to dopaminergic therapies and does not capture other motor or non-motor aspects of APD, while some patients with a low body mass index may never reach a LED dose $>1000 \mathrm{mg} /$ day despite having advanced disease. In a similar approach, Weir and colleagues used LED $>1100 \mathrm{mg} /$ day as one of 
UPDRS score

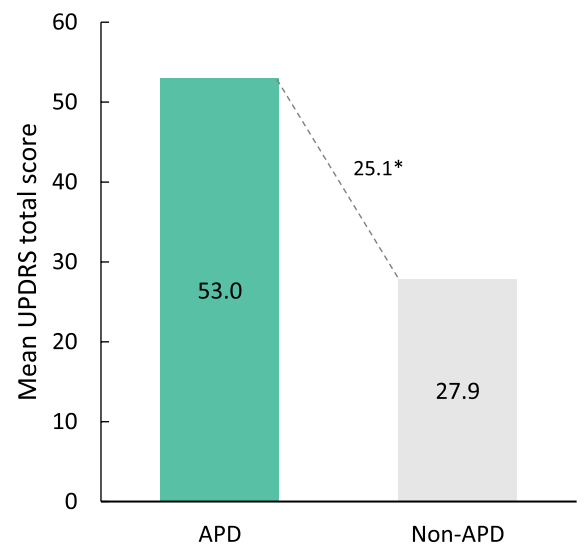

Falls

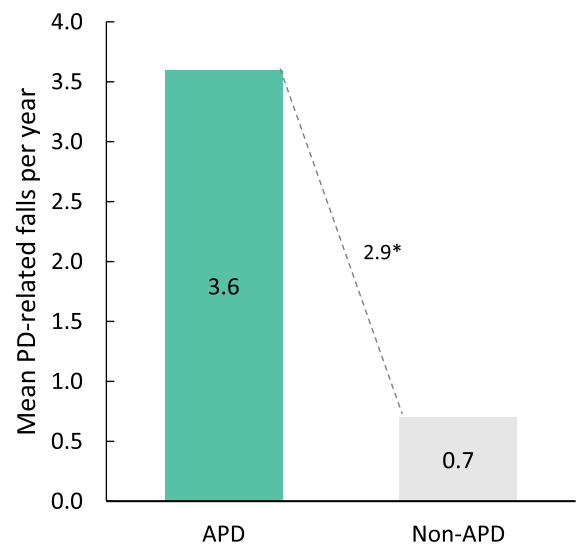

MMSE score

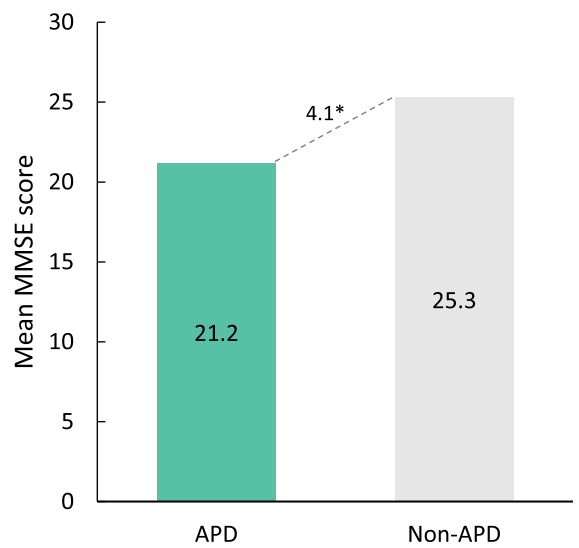

ADL independence

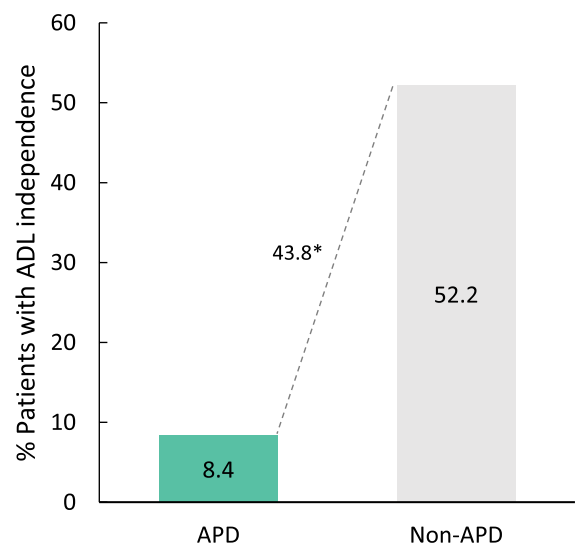

Fig. 2 Measures of clinical burden in APD patients compared with non-APD patients (physician judgement). ${ }^{*} p<0.0001$. ADL, activities of daily living; APD, advanced Parkinson's disease; MMSE, Mini-Mental State Examination; PD, Parkinson's disease; UPDRS, Unified Parkinson's Disease Rating Scale

several proxy markers of APD in a study of PD-related economic costs in the UK, alongside dyskinesias, falls, dementia, psychosis, hospital admission primarily due to PD, and nursing home placement. This study showed an increased economic burden of PD among patients meeting these markers of advanced disease [38].

The results of our study suggest that the 5-2-1 screening criteria would be a useful method for screening patients with advanced disease who may require more tailored assessment and treatment. Having easily identified people with PD who have a greater burden (5-2-1-positive patients), more comprehensive management instruments (i.e., Making Informed Decisions to Aid Timely Management of Parkinson's Disease [MANAGE-PD] tool) [39] can be subsequently adopted to ensure further optimization of treatment. Despite recent advances in the development of screening and management tools, an urgent need remains for a screening tool that is simple to use and remember, is based on readily available information, and that clearly identifies patients with a greater disease burden who may benefit from treatment optimization, including a possible second-line therapy.

The MANAGE-PD is a validated tool designed to support clinician decision-making and facilitate standardized and comprehensive assessment of PD-related symptoms [39]. The 5-2-1 criteria aligns with 3 out of 5 key items assessed by MANAGE-PD section 1 to identify patients with inadequate symptoms control. MANAGEPD section 1 also includes additional questions relating to severity unpredictable fluctuations of motor symptoms and limitations in activities of daily living, making it slightly longer to use, but more comprehensive in its assessment. In a similar way anticipated for the 5-2-1 criteria, MANAGE-PD section 1 groups patients into controlled or inadequately controlled on current therapy. 
Hospitalization rate

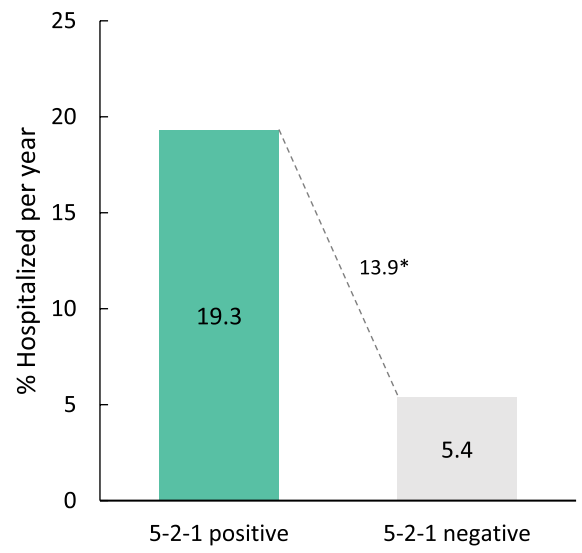

Professional caregiver hours

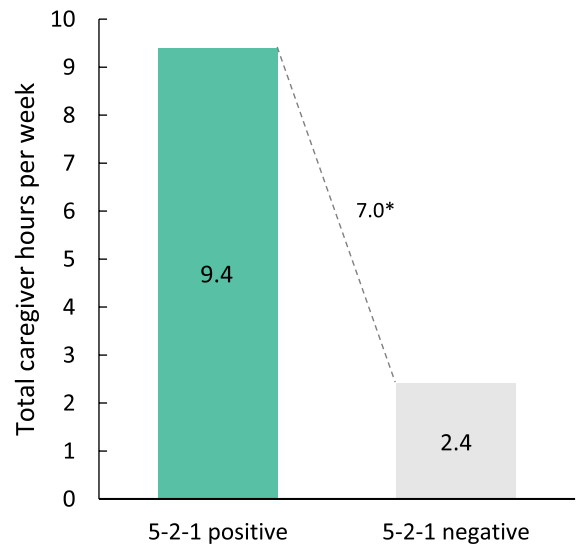

Physician consultations

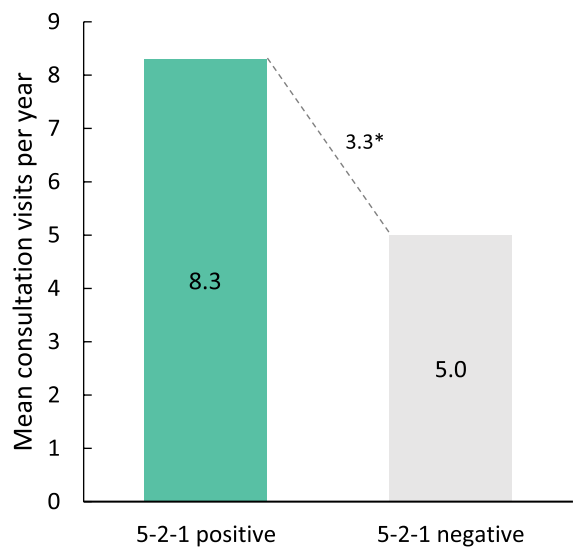

Non-professional caregiver hours

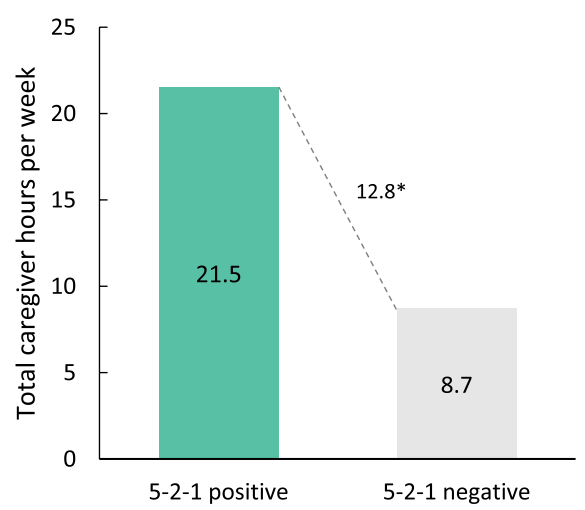

Fig. 3 Measures of healthcare resource utilization in 5-2-1-positive patients compared with 5-2-1-negative patients. ${ }^{*} p<0.0001 .5-2-1$, taking $\geq 5$ doses of oral levodopa/day, OR having $\geq 2 \mathrm{~h}$ 'off'-time/waking day, OR having $\geq 1 \mathrm{~h}$ troublesome dyskinesia/waking day

A recently-reported study compared the 5-2-1 criteria to a newly developed screening tool designed to predict eligibility for referral for DAT evaluation [18]. In this study, a panel of experts assessed 259 consecutive DAT-naive PD patients, of whom 17 were considered eligible for DAT. The study reported several strong predictors of eligibility for referral for DAT, including the presence of response fluctuations and troublesome dyskinesias. Based on their findings, the authors constructed a three-factor screening tool based on LED, response fluctuations, and troublesome dyskinesias, to screen for patients eligible for DAT referral. This study reported a sensitivity and a specificity of 88 and $98 \%$ compared with 94 and $73 \%$, respectively, for the 5-2-1 criteria [18]. The authors note that their tool corroborates the concepts of the 5-2-1 criteria, and the study appears to confirm the validity of the overall approach. In this regard, the 5-2-1 concept has identified a new way to think about DAT-eligible patients and has motivated clinicians to find ways to enhance identification.

One limitation of our study was the acceptance of the physician's opinion of disease severity as the reference against which the 5-2-1 screening criteria were assessed. However, as physician judgement was informed based on the overall condition of the patient, this was considered the best available proxy for management in clinical practice. Furthermore, the categorization of a patient in practice may depend on the experience of the physician, as well as heterogeneous features of PD, including NMS which are not encompassed in 5-2-1. However, although the 5-2-1 screening criteria are driven by MS, there is also a high correlation with other factors, such that patients with advanced or severe MS are also likely to experience NMS [40,41]. It is also worth noting that the rate of potential APD patients identified through the $5-2-1$ screening criteria in this study (33\%) was higher than that identified by physicians (15\%), thus reinforcing 
Hospitalization rate

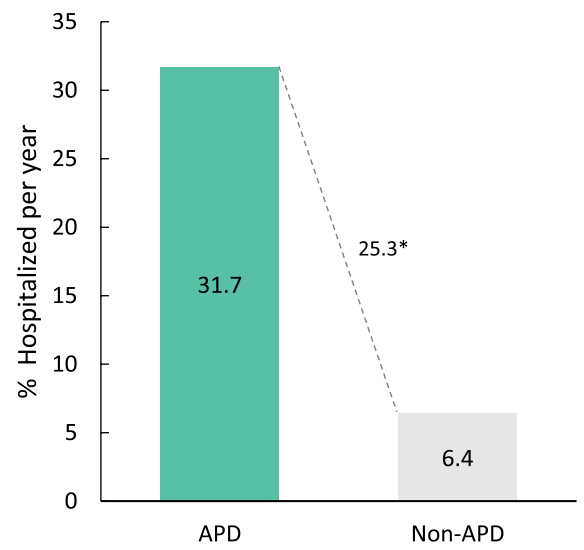

Professional caregiver hours

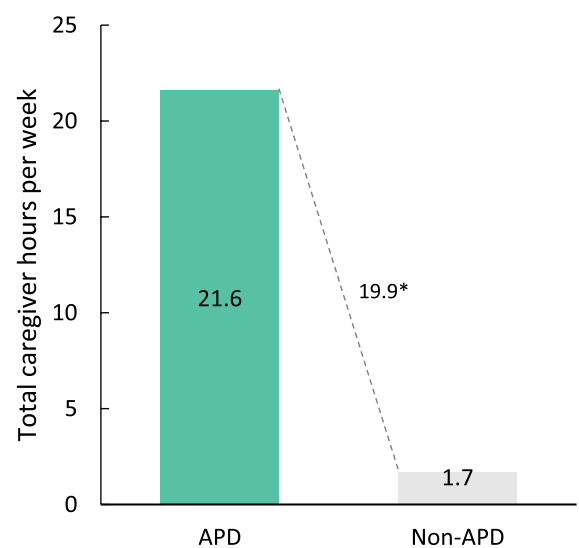

Physician consultations

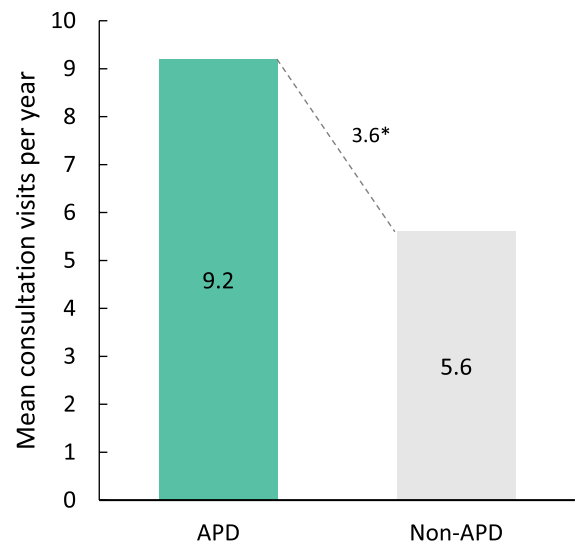

Non-professional caregiver hours

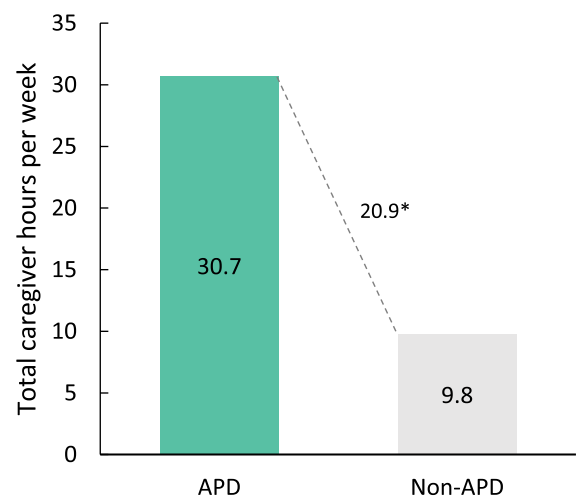

Fig. 4 Measures of healthcare resource utilization in APD patients compared with non-APD patients (physician judgement). ${ }^{*} p<0.0001$. APD, advanced Parkinson's disease

the potential value of the $5-2-1$ criteria as a practical screening tool mandating further patient investigation, rather than a diagnostic tool in its own right. Similar findings were reported by Martinez-Martin et al., where the number of APD patients identified with the CDEPA questionnaire was higher compared to clinician judgment [13]. The CDEPA questionnaire is a screening tool that includes measures of both MS and NMS.

The reliance on patient reports to capture data on falls and number of physician consultations is also a potential limitation of this study. However, although self-report has clear limitations, potential inaccuracies may impact both groups (5-2-1 positive and 5-2-1 negative), while both measures are used as markers of unmet need, rather than for precise quantification. A further limitation of this study is that the only aspects of HCRU considered were hospitalization rate, physician consultations, and amount of caregiver help; meaning that the important contributions of other healthcare professionals such as nurses, physiotherapists and occupational/speech therapists were not captured. In addition, we were not able to evaluate the full economic burden of PD, including indirect costs of treatment and productivity losses. All aspects of the economic burden require further consideration in future studies. Our study also has the limitations inherent in point-in-time studies, including the risk of bias from the absence of randomization and/or due to physician, patient, and caregiver recall limitations. However, this large, international dataset is derived from the Parkinson's DSP, which has been validated for capturing large, statistically robust samples of global real-world evidence, while the data collected reflect current clinical practice. Future studies should evaluate the potential impact of timely escalation in therapy, which may include DAT, to alleviate the disease burden in patients meeting the 5-2-1 screening criteria. 
PD-related quality of life

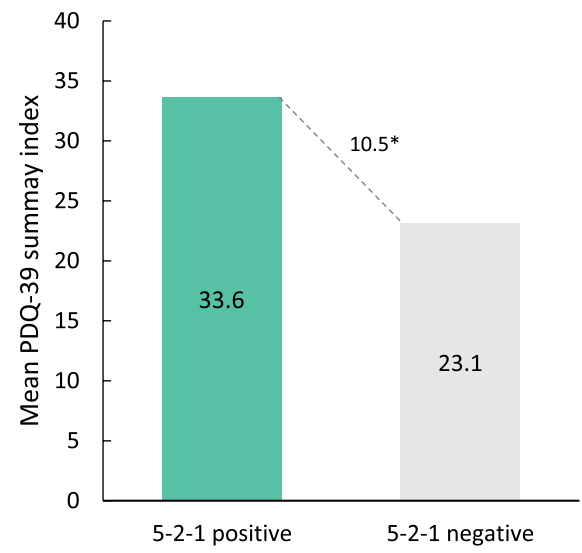

PD treatment satisfaction

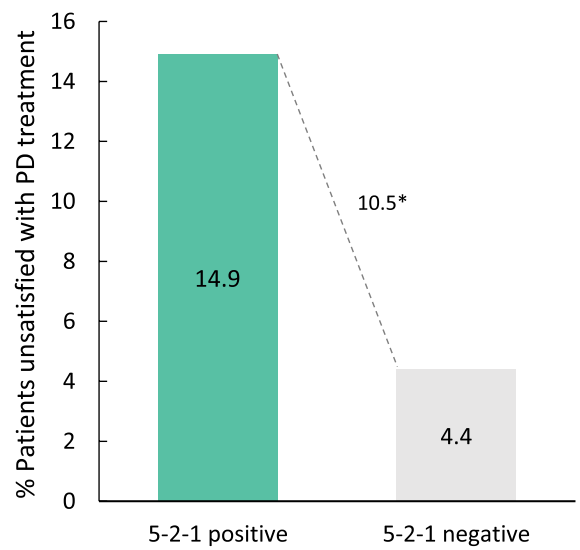

Health-related quality of life

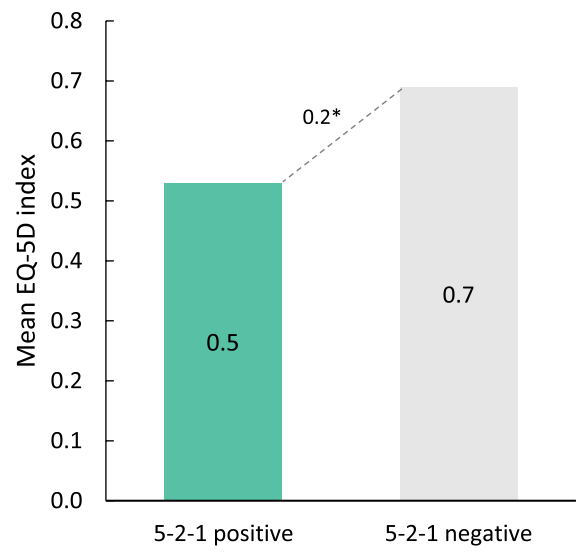

Caregiver burden

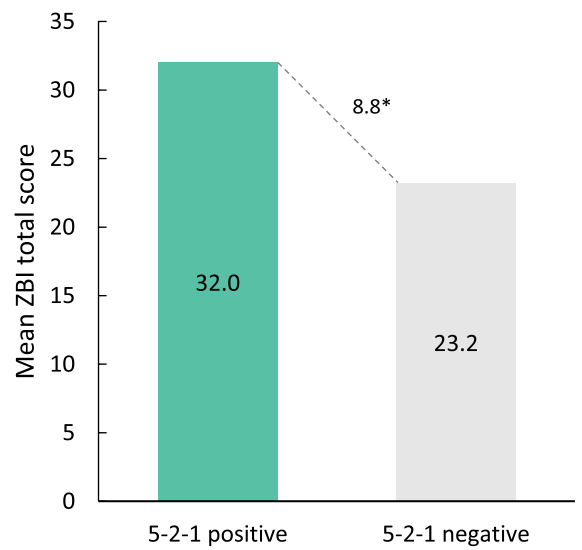

Fig. 5 Measures of humanistic burden in 5-2-1-positive patients compared with 5-2-1-negative patients. ${ }^{*} p<0.0001 .5-2-1$, taking $\geq 5$ doses of oral levodopa/day, OR having $\geq 2$ h 'off'-time/waking day, OR having $\geq 1$ h troublesome dyskinesia/waking day. EQ-5D, EuroQol 5-Dimension (1.0 indicates best QoL); PD, Parkinson's disease; PDQ-39, Parkinson's Disease Questionnaire-39 (higher score indicates worse QoL); ZBI, Zarit Burden Interview

Some consideration should also be given to 'discordant' patients, in whom clinician judgment and 5-2-1 screening criteria did not align. It is interesting that the majority of these patients (21.1\%) were 5-2-1 positive and non-advanced per their physicians, which is consistent with the potential utility of the $5-2-1$ criteria as an early screening tool for advancing PD. In addition, the 5-2-1-negative and clinician-defined advanced group were more likely to have dementia and other cognitive/behavioral symptoms of PD (including confusion, short-term memory loss, difficulties planning, and hallucinations), suggesting that NMS are a significant burden in this patient group. As noted above, NMS are not captured by the 5-2-1 screening criteria, which may explain why a small number of patients whose advanced disease was driven by NMS may have been 5-2-1 negative.

\section{Conclusions}

This study demonstrated the robust screening performance of the 5-2-1 screening criteria in recognizing patients with APD who are sub-optimally controlled on oral PD treatment. This evidence also highlighted the increased clinical, humanistic, and HCRU burden experienced by $5-2-1$ positive patients. In particular, the 5-2-1 screening criteria represent a tool that is simple to use and remember, uses readily available information, and demonstrates clinical value in identifying patients with suboptimal disease control, worse QoL, and higher caregiver burden. The 5-2-1 screening criteria provide an objective and reliable tool that may aid in the timely 
PD-related quality of life

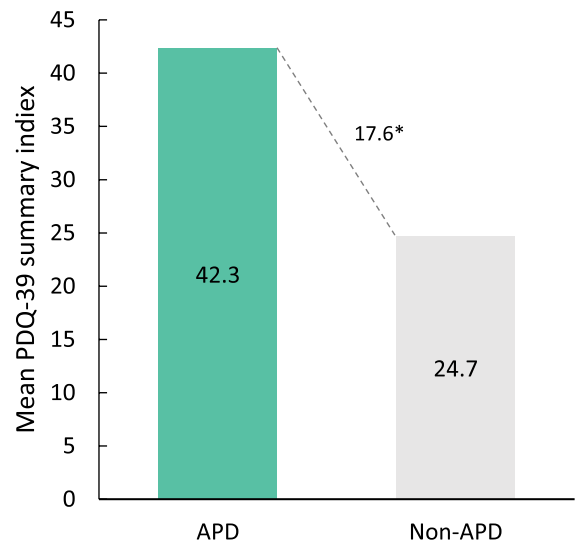

PD treatment satisfaction

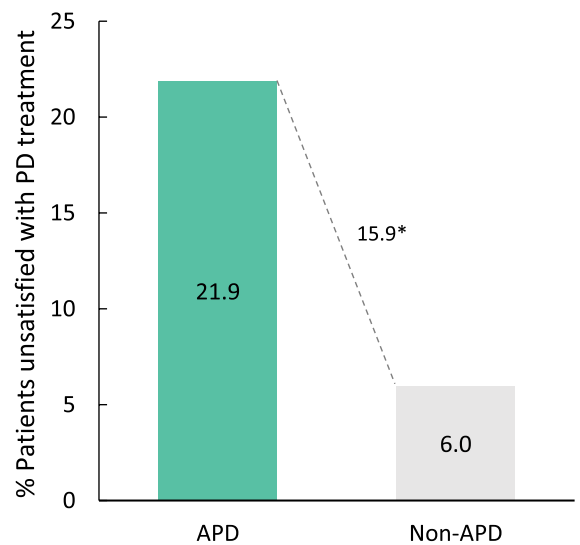

Health-related quality of life

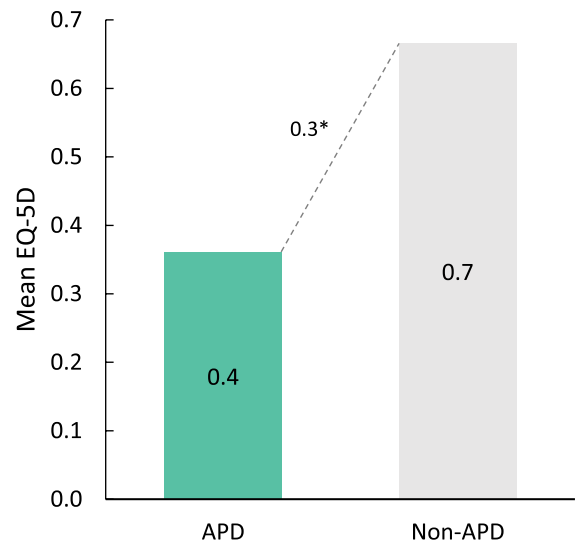

Caregiver burden

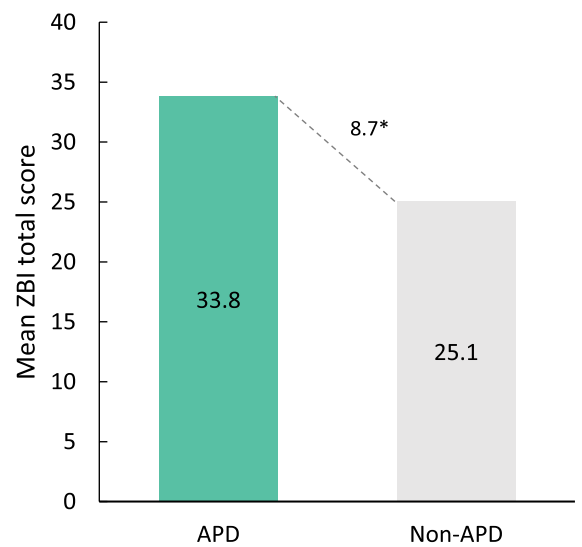

Fig. 6 Measures of humanistic burden in APD patients compared with non-APD patients (physician judgement). ${ }^{*} p<0.0001$. APD, Advanced Parkinson's disease; EQ-5D, EuroQol 5-Dimension (1.0 indicates best QoL); PD, Parkinson's disease; PDQ-39, Parkinson's Disease Questionnaire-39 (higher score indicates worse QoL); ZBI, Zarit Burden Interview

identification and treatment optimization of patients who are inadequately controlled on oral PD medications.

\section{Abbreviations}

ADL: Activities of daily living; APD: Advanced Parkinson's disease; AUC: Area under the curve; Cl: Confidence interval; DAT: Device-aided therapy; DSP: Disease Specific Program ${ }^{\mathrm{TM}}$; EQ-5D: EuroQol 5 Dimension; H\&Y: Hoehn and Yahr scale; LCIG: Levodopa-carbidopa intestinal gel; LED: Levodopa equivalent dose; MANAGE-PD: Making Informed Decisions to Aid Timely Management of Parkinson's Disease; MMSE: Mini-Mental State Examination; MS: Motor symptoms; NMS: Non-motor symptoms; OR: Odds ratio; PD: Parkinson's disease; PDCS: Parkinson Disease Composite Scale; PDQ-39: Parkinson's Disease Questionnaire-39; QoL: Quality of life; SD: Standard deviation; UPDRS: Unified Parkinson's Disease Rating Scale; ZBI: Zarit Burden Interview.

\section{Supplementary Information}

The online version contains supplementary material available at https://doi. org/10.1186/s12883-022-02560-1.

Additional file 1.

\section{Acknowledgments}

We acknowledge Alex Gillespie, James Pike, and Eddie Jones for their support on conducting the data analysis. Medical writing support under the guidance of the authors was provided by Rachel Danks, on behalf of Adelphi Real World and AbbVie, and was funded by AbbVie in accordance with Good Publication Practice (GPP3) guidelines.

\section{Authors' contributions}

IAM, AnA, AIA, and YJ contributed to study conception and design. All authors contributed to results interpretation and manuscript development. All authors read, contributed to, and approved all the drafts and the final manuscript, and therefore meet the criteria for authorship in accordance with the International Committee of Medical Journal Editors (ICMJE) guidelines. In addition, all named authors take responsibility for the integrity of the work as a whole, and have given their approval for this version to be published.

\section{Funding}

Data collection was undertaken by Adelphi Real World as part of an independent survey, entitled the Adelphi Parkinson's Disease Specific Programme, sponsored by multiple pharmaceutical companies of which one was AbbVie. AbbVie did not influence the original survey through either contribution to the design of questionnaires or data collection. The study described here 
using data from the Adelphi Parkinson's Disease Specific Programme was funded by AbbVie.

\section{Availability of data and materials}

The datasets generated and/or analyzed during the current study are not publicly available as they are the intellectual property of Adelphi Real World but are available from the corresponding author on reasonable request.

\section{Declarations}

\section{Ethics approval and consent to participate}

The Disease Specific Programme was conducted in accordance with the European Pharmaceutical Market Research Association (EphMRA) Code of Conduct. The study protocol (reference number AG8689) was submitted to the Western Institutional Review Board, and an ethics waiver was provided as it was determined that ethics approval was not required for this study. All data were collected following procedures with ethics committee approval, and data were fully de identified prior to receipt by Adelphi Real World. The respondents provided informed consent for use of their anonymized and aggregated data for research and publication in scientific journals. All data, i.e. methodology, materials, data, and data analysis, that support the findings of this survey are the intellectual property of Adelphi Real World. As such no administrative permissions were required to access and use the data.

\section{Consent for publication}

Not applicable.

\section{Competing interests}

IAM has participated in research funded by Parkinson Foundation, Tourette Association of America, Dystonia Coalition, AbbVie, Biogen, Boston Scientific, Eli Lilly, Impax, Neuroderm, Prilenia, Revance, and Teva but has no owner interest in any pharmaceutical company. She has received travel compensation or honoraria from Cleveland Clinic, Parkinson Foundation, Tourette Association, and International Association of Parkinsonism and Related Disorders and royalties from Robert Rose Publishers; PMM has received honoraria from Editorial Viguera and Takeda Pharmaceuticals for lecturing in courses; from Britannia for writing an article in their Parkinson's Disease Medical JournalKinetic; and from the International Parkinson and Movement Disorder Society (MDS) for management of the Program on Rating Scales. Grants from the MDS for development and validation of the MDS-NMS; SAJ has no relevant disclosures; KRC has received educational funding from UCB, and honoraria for sponsored symposiums from UCB, AbbVie, Britannia, US Worldmeds, Otsuka, Medtronic, Zambon and acted as a consultant for AbbVie, UCB, Britannia, Medtronic and Mundipharama; JJ-S has received consulting fees from Teva, Nuvelution, Bracket, Amneal, Revance, AbbVie, CNS Ratings, St. Jude Medical, Medtronic and research funding from Impax; PO has received compensations for consultancy and speaker related activities from AbbVie, Bial, Britannia, Ever Pharma, Lobsor, Nordic Infucare, Stada, and Zambon. Odin has received royalties from Uni-Med Verlag; MS received speakers' honoraria and/or consultation fees from AbbVie, Boston Scientific, Desitin, Ever Pharma, Krka, Medtronic and Sandoz, he received support from the IBM and Slovak grant agencies under contracts APV-18-0547 and VEGA 1/0596/19 and the Operational Programme Integrated Infrastructure, funded by the ERDF under No. ITMS2014+:313011 V455; MJS has received honoraria and/or consultation fees from AbbVie, Abbott, Acorda Therapeutics, Amneal, Teva, Sunovion, Neurocrine, Medtronic, and Merz. He has also received research compensation from Cerevel and AbbVie; SL declares no relevant conflict of interest to present study; JD declares no relevant conflict of interest to present study; AIA, YJJ, $\mathrm{PLK}, \mathrm{KO}, \mathrm{LB}, \mathrm{SF}$, and MYL are employees of AbbVie and may own stocks/shares in the company; JW is an employee of Adelphi Real World, a consulting company that was hired by AbbVie to perform analyses on the Adelphi Disease Specific Programme database; AnA has received compensation for consultancy and speaker related activities from UCB, Boehringer Ingelheim, Britannia, AbbVie, Kyowa Kirin, Zambon, Bial, Neuroderm, Theravance Biopharma, Roche; he receives research support from Chiesi Pharmaceuticals, Lundbeck, Horizon 2020 - Grant 825785, Horizon2020 Grant 101016902, Ministry of Education University and Research (MIUR) Grant ARS01_01081, Cariparo Foundation. He serves as consultant for Boehringer Ingelheim for legal cases on pathological gambling.

\begin{abstract}
Author details
${ }^{1}$ University of Florida, Fixel Institute for Neurological Diseases, Gainesville, FL, USA. ${ }^{2}$ Center for Networked Biomedical Research in Neurodegenerative Diseases (CIBERNED), Carlos III Institute of Health, Madrid, Spain. ${ }^{3}$ Parkinson Foundation Centre of Excellence, King's College Hospital and King's College, London, UK. ${ }^{4}$ University of Lund, Lund, Sweden. ${ }^{5}$ Department of Neurology, P. J. Šafárik University, Košice, Slovakia. ${ }^{6}$ Department of Neurology, University Hospital of L. Pasteur, Košice, Slovakia. ${ }^{7}$ Department of Neurology, Icahn School of Medicine at Mount Sinai, New York, NY, USA. ${ }^{8}$ Texas Movement Disorder Specialists, Georgetown, TX, USA. ${ }^{9}$ European Parkinson's Disease Association (EPDA), Sevenoaks, UK. ${ }^{10} \mathrm{Grupo}$ de patologia médica, nutrição e exercício clínico (PaMNEC) do CiiEM, Almada, Portugal. ${ }^{11}$ Parkinson \& Movement Disorder Alliance, Tucson, USA. ${ }^{12}$ AbbVie Inc., North Chicago, IL, USA. ${ }^{13}$ University of Illinois at Chicago, Chicago, IL, USA. ${ }^{14}$ Adelphi Real World, Bollington, UK. ${ }^{15}$ Parkinson and Movement Disorders Unit, Department of Neuroscience, University of Padua, Padova, Italy.
\end{abstract}

Received: 26 July 2021 Accepted: 19 January 2022

Published online: 24 January 2022

\section{References}

1. Dorsey ER, Sherer T, Okun MS, Bloem BR. The emerging evidence of the Parkinson pandemic. J Parkinsons Dis. 2018;8(s1):S3-8.

2. GBD 2015 Neurological Disorders Collaborator Group. Global, regional, and national burden of neurological disorders during 1990-2015: a systematic analysis for the Global Burden of Disease Study 2015. Lancet Neurol. 2017;16(11):877-97.

3. Dahodwala N, Pettit AR, Jahnke J, Li P, Ladage VP, Kandukuri PL, et al. Use of a medication-based algorithm to identify advanced Parkinson's disease in administrative claims data: associations with claims-based indicators of disease severity. Clin Parkinsonism Relat Disord. 2020;3:100046.

4. Antonini A, Stoessl AJ, Kleinman LS, Skalicky AM, Marshall TS, Sail KR, et al. Developing consensus among movement disorder specialists on clinical indicators for identification and management of advanced Parkinson's disease: a multi-country Delphi-panel approach. Curr Med Res Opin. 2018;34(12):2063-73.

5. Marrinan S, Emmanuel AV, Burn DJ. Delayed gastric emptying in Parkinson's disease. Mov Disord. 2014;29(1):23-32.

6. Kulisevsky J, Luquin MR, Arbelo JM, Burguera JA, Carrillo F, Castro A, et al. Advanced Parkinson's disease: clinical characteristics and treatment. Part II. Neurologia. 2013;28(9):558-83.

7. Dietrichs E, Odin P. Algorithms for the treatment of motor problems in Parkinson's disease. Acta Neurol Scand. 2017;136(5):378-85.

8. Odin P, Ray Chaudhuri K, Slevin JT, Volkmann J, Dietrichs E, MartinezMartin $\mathrm{P}$, et al. Collective physician perspectives on non-oral medication approaches for the management of clinically relevant unresolved issues in Parkinson's disease: consensus from an international survey and discussion program. Parkinsonism Relat Disord. 2015;21(10):1133-44.

9. Lerche S, Heinzel S, Alves GW, Barone P, Behnke S, Ben-Shlomo Y, et al. Aiming for study comparability in Parkinson's disease: proposal for a modular set of biomarker assessments to be used in longitudinal studies. Front Aging Neurosci. 2016;8:121.

10. Titova N, Martinez-Martin P, Katunina E, Chaudhuri K. Advanced Parkinson's or "complex phase" Parkinson's disease? Re-evaluation is needed. J Neural Transm (Vienna). 2017;124(12):1529-37.

11. Hoehn MM, Yahr MD. Parkinsonism: onset, progression and mortality. Neurology. 1967;17(5):427-42.

12. Martinez-Martin P, Radicati FG, Rodriguez Blazquez C, Wetmore J, Kovacs $\mathrm{N}$, Ray Chaudhuri K, et al. Extensive validation study of the Parkinson's disease composite scale. Eur J Neurol. 2019;26(10):1281-8.

13. Martinez-Martin P, Kulisevsky J, Mir P, Tolosa E, García-Delgado P, Luquin MR. Validation of a simple screening tool for early diagnosis of advanced Parkinson's disease in daily practice: the CDEPA questionnaire. NPJ Parkinsons Dis. 2018;4:20.

14. Goetz CG, Poewe W, Rascol O, Sampaio C, Stebbins GT, Counsell C, et al. Movement Disorder Society task force report on the Hoehn and Yahr staging scale: status and recommendations. Mov Disord. 2004;19(9):1020-8. 
15. Luquin MR, Kulisevsky J, Martinez-Martin P, Mir P, Tolosa ES. Consensus on the definition of advanced Parkinson's disease: a neurologists-based Delphi study (CEPA study). Parkinsons Dis. 2017;2017:4047392.

16. Santos-García D, de Deus FT, Suárez Castro E, Aneiros Díaz A, McAfee D. 5-2-1 Criteria: A Simple Screening Tool for Identifying Advanced PD Patients Who Need an Optimization of Parkinson's Treatment. Parkinsons Dis. 2020;2020:7537924.

17. Fasano A, Fung VSC, Lopiano L, Elibol B, Smolentseva IG, Seppi K, et al. Characterizing advanced Parkinson's disease: OBSERVE-PD observational study results of 2615 patients. BMC Neurol. 2019;19(1):50.

18. Moes HR, ten Kate J, Portman AT, van Harten B, van Kesteren ME, Mondria T, et al. Timely referral for advanced therapy in Parkinson's disease. Development of a screening tool. medRxiv. 2021:2021.02.10.21251496. https:// www.medrxiv.org/content/10.1101/2021.02.10.21251496v1.

19. Aldred J, Anca-Herschkovitsch M, Antonini A, Bajenaru O, Bergmann L, Bourgeois $\mathrm{P}$, et al. Application of the '5-2-1'screening criteria in advanced Parkinson's disease: interim analysis of DUOGLOBE. Neurodegener Dis Manag. 2020;10(5):309-23.

20. Anderson P, Benford M, Harris N, Karavali M, Piercy J. Real-world physician and patient behaviour across countries: disease-specific Programmes - a means to understand. Curr Med Res Opin. 2008;24(11):3063-72.

21. Movement Disorder Society Task Force on Rating Scales for Parkinson's Disease. The unified Parkinson's disease rating scale (UPDRS): status and recommendations. Mov Disord. 2003;18(7):738-50.

22. Tombaugh TN, McIntyre NJ. The mini-mental state examination: a comprehensive review. J Am Geriatr Soc. 1992;40(9):922-35.

23. Charlson ME, Pompei P, Ales KL, MacKenzie CR. A new method of classifying prognostic comorbidity in longitudinal studies: development and validation. J Chronic Dis. 1987;40(5):373-83.

24. Peto V, Jenkinson C, Fitzpatrick R, Greenhall R. The development and validation of a short measure of functioning and well being for individuals with Parkinson's disease. Qual Life Res. 1995;4(3):241-8.

25. Devlin NJ, Brooks R. EQ-5D and the EuroQol group: past, present and future. Appl Health Econ Health Policy. 2017;15(2):127-37.

26. Zarit SH, Reever KE, Bach-Peterson J. Relatives of the impaired elderly: correlates of feelings of burden. Gerontologist. 1980;20(6):649-55.

27. Fischer JE, Bachmann LM, Jaeschke R. A readers' guide to the interpretation of diagnostic test properties: clinical example of sepsis. Intensive Care Med. 2003;29(7):1043-51.

28. Greiner M, Pfeiffer D, Smith RD. Principles and practical application of the receiver-operating characteristic analysis for diagnostic tests. Prev Vet Med. 2000;45(1-2):23-41.

29. Swets JA. Measuring the accuracy of diagnostic systems. Science. 1988;240(4857):1285-93.

30. Antonini A, Isaias IU, Rodolfi G, Landi A, Natuzzi F, Siri C, et al. A 5-year prospective assessment of advanced Parkinson disease patients treated with subcutaneous apomorphine infusion or deep brain stimulation. J Neurol. 2011;258(4):579-85.

31. Simuni T, Luo ST, Chou KL, Fernandez H, He B, Parashos S. Rankin scale as a potential measure of global disability in early Parkinson's disease. J Clin Neurosci. 2013;20(9):1200-3.

32. Pistacchi M, Gioulis M, Sanson F, De Giovannini E, Filippi G, Rossetto F, et al. Gait analysis and clinical correlations in early Parkinson's disease. Funct Neurol. 2017;32(1):28-34.

33. Politis M, Wu K, Molloy S. P GB, Chaudhuri KR, Piccini P. Parkinson's disease symptoms: the patient's perspective. Mov Disord. 2010;25(11):1646-51.

34. Lökk J. Lack of information and access to advanced treatment for Parkinson's disease patients. J Multidiscip Healthc. 2011;4:433-9.

35. Chen H, Zhao EJ, Zhang W, Lu Y, Liu R, Huang X, et al. Meta-analyses on prevalence of selected Parkinson's nonmotor symptoms before and after diagnosis. Transl Neurodegener. 2015;4(1):1.

36. Hauser RA, Lyons KE, Pahwa R. The UPDRS-8: a brief clinical assessment scale for Parkinson's disease. Int J Neurosci. 2012;122(7):333-7.

37. Titova N, Qamar MA, Chaudhuri KR. Biomarkers of Parkinson's disease: an introduction. Int Rev Neurobiol. 2017;132:183-96.

38. Weir S, Samnaliev M, Kuo TC, Tierney TS, Walleser Autiero S, Taylor RS, et al. Short- and long-term cost and utilization of health care resources in Parkinson's disease in the UK. Mov Disord. 2018;33(6):974-81.

39. Antonini A, Odin P, Schmidt P, Cubillos F, Standaert DG, Henriksen T, et al. Validation and clinical value of the MANAGE-PD tool: a clinician-reported tool to identify Parkinson's disease patients inadequately controlled on oral medications. Parkinsonism Relat Disord. 2021;92:59-66.

40. Schapira AHV, Chaudhuri KR, Jenner P. Non-motor features of Parkinson disease. Nat Rev Neurosci. 2017;18(7):435-50.

41. Goldman JG, Postuma R. Premotor and nonmotor features of Parkinson's disease. Curr Opin Neurol. 2014;27(4):434-41.

\section{Publisher's Note}

Springer Nature remains neutral with regard to jurisdictional claims in published maps and institutional affiliations.
Ready to submit your research? Choose BMC and benefit from:

- fast, convenient online submission

- thorough peer review by experienced researchers in your field

- rapid publication on acceptance

- support for research data, including large and complex data types

- gold Open Access which fosters wider collaboration and increased citations

- maximum visibility for your research: over 100M website views per year

At BMC, research is always in progress.

Learn more biomedcentral.com/submissions 